\title{
A novel PLC channel modeling method and channel characteristic analysis of a smart distribution grid
}

\author{
Jian Le ${ }^{1 *}$, Cao Wang ${ }^{1}$, Wu Zhou' ${ }^{1}$ Yong-yan Liu' and Wei Cai ${ }^{2}$
}

\begin{abstract}
Power line carrier (PLC) technology plays an increasingly important role in the realization of cost-effective communication in a smart distribution grid. No current channel modeling method is universally applicable to more complex topologies that may emerge in smart grids, such as ring and mesh topologies. This paper presents a novel PLC channel modeling method based on the information node concept, and the universality and feasibility of the proposed method are demonstrated with applications in modeling networks with ring and mesh topologies. The factors that affect the channel characteristics of the networks and the laws that govern their behaviors for different types of topologies are analyzed. The validity and effectiveness of the proposed method are proven using simulation and laboratory tests. This paper provides the necessary theoretical basis and technical means to design the PLC modulation method for smart distribution grids.
\end{abstract}

Keywords: Smart distribution grid, Transfer matrix, Power-line communication, Information nodes, Channel characteristics

\section{Introduction}

Reliable and cost-effective communication networks are the basis and premise for the realization of Smart Grids. The power line carrier (PLC) technology [1-3], which has been applied in traditional power systems to transfer voice or data at high speed over the power line with a power flow, is considered a suitable candidate [4, 5]. However, the channel of the PLC has high-frequency selectivity with complex signal attenuation because of the poor working condition, serious interference, and large time variation. As a result, numerous studies have considered the power-line channel modeling method to accurately establish an effective PLC transmission model and select the best transmission frequency according to the frequency selectivity transmission characteristic [6-8].

The existing channel modeling methods can be divided into time-domain [9-12] and frequency-domain types [13-18]. Time-domain methods, which can be further divided into top-down $[9,10]$ and bottom-up $[11,12]$ types according to the approach used to obtain the necessary parameters, treat the PLC channel as a multi-path

\footnotetext{
* Correspondence: lej01@mails.tsinghua.edu.cn

${ }^{1}$ School of Electrical Engineering, Wuhan University, Wuhan 430072, China

Full list of author information is available at the end of the article
}

environment and use the reflection model to represent the physical characteristics of the channel. The top-down method regards the PLC channel as a multi-path model and uses curve fitting techniques to obtain the required parameters based on the actual measurement results of the transmission characteristics. Various curve fitting techniques are used, and the complexity and time variation of the PLC channels cause large differences between measurement results. Hence, the models obtained by different researchers are notably different, and none can guarantee the best performance under all operation conditions of the network. The main principle of the bottom-up methods is to consider the reflection and attenuation at the impedance mismatch points and subsequently enumerate, simplify, and classify all paths between the signal transmitter and the information receiver based on the actual network topology. This type of method simplifies the forward traveling wave to such an extent that it results in large errors. The method is also computationally expensive and can only be applied to the tree topology.

To calculate the transmission characteristics of the entire network, frequency-domain methods break the entire network into several cascaded sub-networks and calculate the transmission matrix [13-15] or scattering matrix [16-18] for each sub-network. This type of method has the 
advantage of considering the reflective properties of all signals regardless of the network complexity. However, it cannot be easily applied in the ring and mesh topologies, which may emerge in smart distribution grids, because these topologies cannot be broken into sub-networks. H. Meng [18] derived a channel model method for a ring topology using scattering parameters; however, this method has difficulty considering multiple rings and mesh topologies.

A new modeling method to analyze the transfer characteristics of the PLC channel of a network with arbitrary topology is proposed in this paper, and its application in the network with a ring or mesh topology is demonstrated in detail [19]. The remainder of this paper is structured as follows. In Section II, the principle of the channel modeling method based on the information node concept is presented. Section III shows the application of the proposed method in a network with ring topology; the validity and effectiveness of this method is proven by simulation and laboratory test. The influence laws that govern the channel characteristics of a network with complex mesh topology are analyzed by simulation and laboratory test results in Section V. Finally, in Section VI, the main findings of this paper are summarized.

\section{Channel modeling approach based on information node (Methods)}

\subsection{Network topology and information nodes}

Figure 1 illustrates the topology of a general complex PLC communication network, which is typically composed of signal transmitters, signal receivers, power lines, and network nodes. The purpose of power line channel modeling is to determine the transmission characteristic between a signal transmitter and one of multiple receivers at a given time. The network nodes are divided into internal-type nodes and termination-type nodes. The internal nodes only connect to power lines, such as $C_{m}$ and $C_{n}$ in Fig. 1, and the termination nodes connect to at least one of the loads or the signal source, such as $T_{1}, T_{2}, T_{i}$, and $T_{j}$ in Fig. 1. $V_{S}$ and $Z_{S}$ are the amplitude and impedance of the signal source, respectively.

In this paper, a new concept called the "information node", which can cover important information regarding the node voltage and current, is introduced to help describe the state of the network. Several information nodes are placed at the points as closely as possible around a network node. For example, $D_{t 11}$ and $D_{t 12}$ are the information nodes related to the signal source impedance $Z_{s}$ and power line Line_1, respectively, which connect to termination node $T_{1}$, and $D_{c n 1}$ is the information node of power line Line_k, which connects to internal node $C_{n}$. Thus, at least two relevant information nodes should be assigned to a network node except for the signal source, where the voltages of the signal source and at information node $D_{t 11}$ are different because of the internal impedance $Z_{S}$. Therefore, an additional information node $D_{s}$ should be introduced between the signal source and $D_{t 11}$, as shown in Fig. 1.

By placing all necessary information nodes into the network, the problem of determining the transmission characteristic between signal source $V_{\mathrm{S}}$ and each receiver is converted into a problem of determining the transmission characteristic between information node $D_{S}$ and the information node related to each receiver (e.g., $D_{t 22}$ if the receiver is located at node $T_{2}$ ).

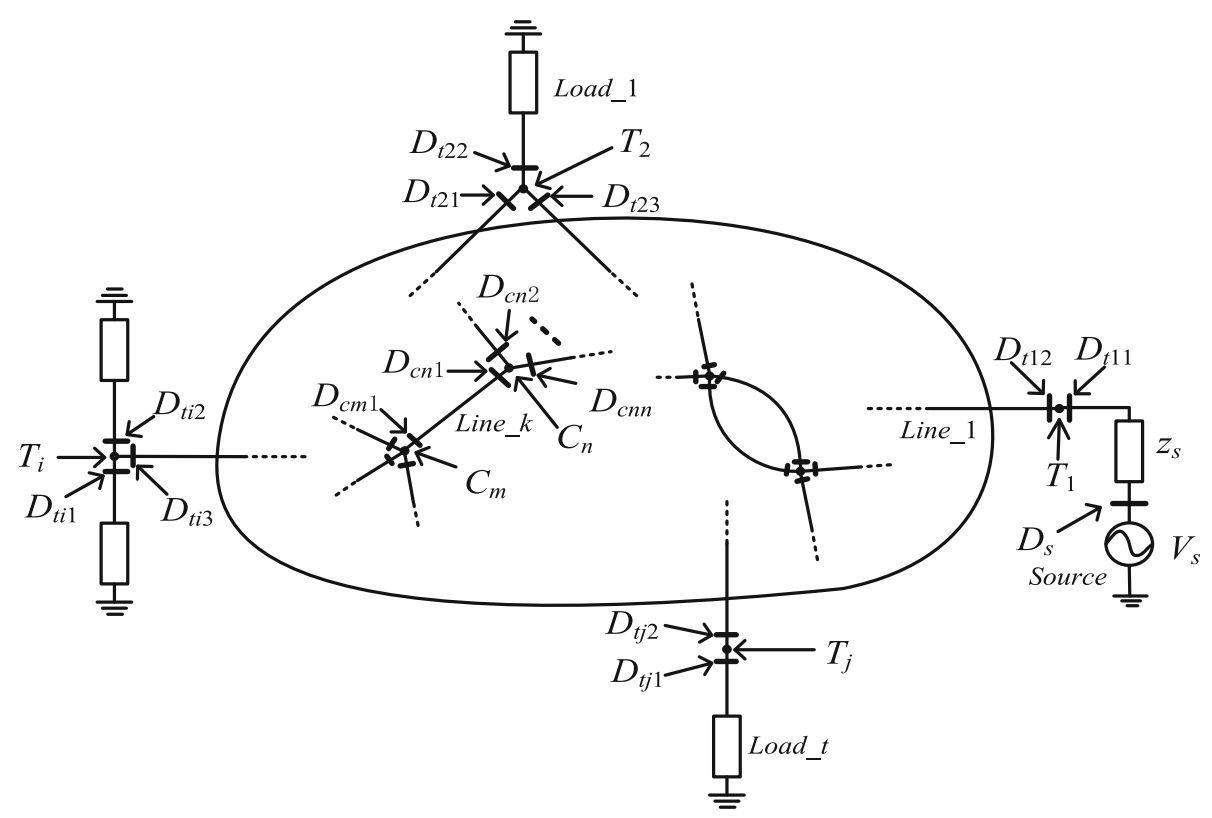

Fig. 1 Complex network topology 


\subsection{Network equations based on information nodes}

Without loss of generality, we assume that there are $m$ power lines and $n$ loads in the power network in Fig. 1. According to the principle of setting the information nodes, $2 m$ and $n$ information nodes are respectively linked with $m$ power lines and $n$ information nodes because a power line must be connected between two information nodes, whereas a load is always related to only one information node. Because the special information nodes $D_{S}$ and $D_{t 11}$ are related to the signal source, the total number $S$ of information nodes in the network is:

$$
S=2 m+n+2
$$

Obviously, the voltage of and the current flowing out of an information node are two unknown quantities. Thus, there are $4 m+2 n+4$ unknown quantities for the entire network. In this paper, the reference direction of the node current is out of the node.

Figure 2 shows the power line Line_k and its two information nodes $D_{c m 1}$ and $D_{c n 1}$.

According to transmission theory, the $V-I$ relationships between information nodes $D_{c m 1}$ and $D_{c n 1}$ are:

$$
\left.\begin{array}{l}
U_{c n 1}=U_{c m 1} \cdot \operatorname{ch}(\gamma x)-I_{c m 1} \cdot Z_{C} \cdot \operatorname{sh}(\gamma x) \\
-I_{c n 1}=I_{c m 1} \cdot \operatorname{ch}(\gamma x)-\frac{U_{c m 1}}{Z_{C}} \operatorname{sh}(\gamma x)
\end{array}\right\}
$$

where $x$ is the length of the power line Line_k, and $\gamma$ and $Z_{C}$ are the propagation constant and characteristic impedance of this power line, respectively. We can list two equations similar to Eq. (2) for each power line; therefore, $2 \mathrm{~m}$ equations can be obtained for $m$ power lines.

For Load_1 in Fig. 1, its voltage $U_{t 22}$ and current $I_{t 22}$ must satisfy Ohm's Law, i.e.,

$$
U_{t 22}=Z_{L 1} \times I_{t 22}
$$

where $Z_{L 1}$ is the load impedance of Load_1. Thus, $n$ equations can be obtained for $n$ loads.

Suppose that there are $k$ information nodes that are related to a network node $C_{n}$, as shown in Fig. 3 .

According to the basic circuit theory, we can obtain $k$ independent equations for this network node as:

$$
\left.\begin{array}{c}
U_{c n 1}=U_{c n 2}=\cdots=U_{c n k} \\
I_{c n 1}+I_{c n 2}+\cdots+I_{c n k}=0
\end{array}\right\}
$$

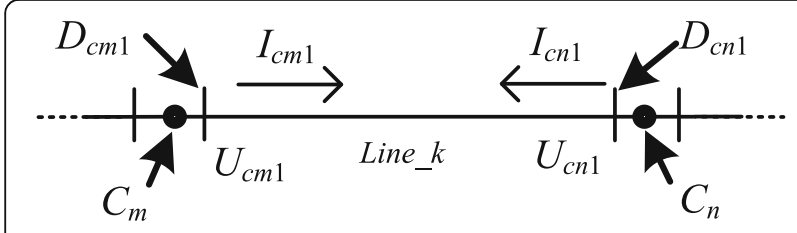

Fig. 2 Power line and its information nodes

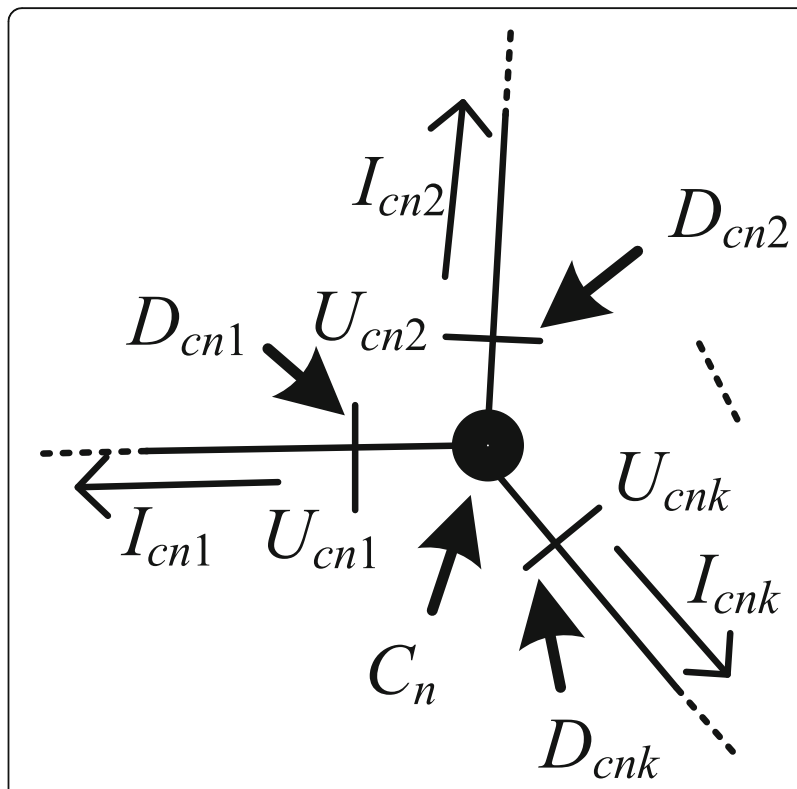

Fig. 3 Network node and its information nodes

For a network with $m$ power lines, $n$ loads, and one signal source, there must be $2 m+n+1$ information nodes, and each node is related to one and only one of the network nodes ( $D s$ is excluded). Thus, $2 m+n+1$ independent equations can be listed for all network nodes.

As shown in Fig. 4, for information nodes $D_{t 11}$ and $D_{S}$, which are related to signal source $V_{S}$, the following two equations are deduced:

$$
\left.\begin{array}{rl}
U_{S} & =U_{t 11}-I_{t 11} \times Z_{S} \\
I_{S} & =-I_{t 11}
\end{array}\right\}
$$

where $Z_{S}$ is the internal impedance of signal source $V_{S}$.

Therefore, for a power network of $m$ power lines and $n$ loads, $2 m+n+2 m+n+1+2=4 m+2 n+3$ independent equations can be obtained based on the above derivations. These equations can be expressed in matrix form as follows:

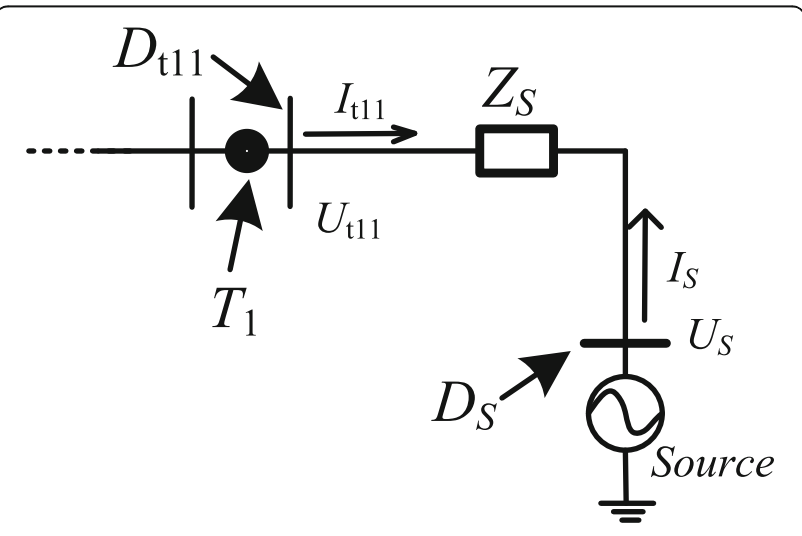

Fig. 4 Signal source and its information nodes 


$$
\left[\begin{array}{cccc}
a_{(1)(1)} & a_{(1)(2)} & \ldots & a_{(1)(4 m+2 n+4)} \\
a_{(2)(1)} & a_{(2)(2)} & \ldots & a_{(2)(4 m+2 n+4)} \\
\vdots & \vdots & \ldots & \vdots \\
a_{(4 m+2 n+3)(1)} & a_{(4 m+2 n+3)(2)} & a_{(4 m+2 n+3)(4 m+2 n+4)}
\end{array}\right]\left[\begin{array}{l}
U_{S} \\
U_{1} \\
\vdots \\
U_{2 m+n+1} \\
I_{S} \\
I_{1} \\
\vdots \\
I_{2 m+n+1}
\end{array}\right]=\left[\begin{array}{c}
0 \\
0 \\
\vdots \\
0 \\
0 \\
0 \\
\vdots \\
0
\end{array}\right]
$$

Supposing the coefficient matrix of Eq. (6) is $A$, we can know that all elements in $A$ are only related to the network topology and component parameters. Because the $4 m+2 n+3$ equations are independent, the rank of coefficient matrix $A$ is

$$
R(A)=4 m+2 n+3
$$

As mentioned, there are $4 m+2 n+4$ unknown variables for the entire network, whereas there are $4 m+2 n+3$ independent equations in the homogeneous linear equations. Therefore, the ratio of any two unknown variables can be calculated easily by solving Eq. (6). Thus, the voltage transmission characteristics between the signal transmitter and the receiver are:

$$
H_{i}(f)=\frac{U_{Z i}}{U_{S}}
$$

where $U_{Z i}$ is the voltage of the information node related to Load_i.

By solving Eq. (6), the theoretical expression for the voltage transmission characteristic between the signal transmitter and the receiver can be easily calculated using a conventional software such as MATLAB. Then, the key factors that affect the channel transmission characteristic and the influence law can be conveniently analyzed. This method is applicable to a network with arbitrary topology.

There are few ring topologies and no mesh topology in smart grids till now. However, in IEEE1547, part 4.1.4 "Distributed resources on distribution secondary grid and spot networks" proposes ring topologies and mesh topologies in distribution networks in the future with distributed generation accessed. In this situation, the proposed method will be able to solve the problems that there is no effective channel modeling method for the application of PLC in a network with ring and mesh topologies.

\section{Channel modeling and laboratory test of a simple ring topology (Results and discussion 1)}

A simple network with a ring topology in Fig. 5 is considered the example to illustrate the application of the modeling method in Section II. There are seven information nodes in Fig. 5 , i.e., $D_{1}$ to $D_{6}$ and $D_{S}$.

Each information node has two unknown quantities. Thus, there are 14 unknown variables for the entire network of 13 independent equations, which can be expressed as:

$\left[\begin{array}{cccccccccccccc}1 & -1 & 0 & 0 & 0 & 0 & 0 & 0 & Z_{S} & 0 & 0 & 0 & 0 & 0 \\ 0 & 0 & 0 & 0 & 0 & 0 & 0 & 1 & 1 & 0 & 0 & 0 & 0 & 0 \\ 0 & 0 & -a_{1} & 0 & 1 & 0 & 0 & 0 & 0 & b_{1} & 0 & 0 & 0 & 0 \\ 0 & 0 & -c_{1} & 0 & 0 & 0 & 0 & 0 & 0 & d_{1} & 0 & 1 & 0 & 0 \\ 0 & 0 & 0 & -a_{2} & 0 & 1 & 0 & 0 & 0 & 0 & b_{2} & 0 & 0 & 0 \\ 0 & 0 & 0 & -c_{2} & 0 & 0 & 0 & 0 & 0 & 0 & d_{2} & 0 & 1 & 0 \\ 0 & 1 & -1 & 0 & 0 & 0 & 0 & 0 & 0 & 0 & 0 & 0 & 0 & 0 \\ 0 & 1 & 0 & -1 & 0 & 0 & 0 & 0 & 0 & 0 & 0 & 0 & 0 & 0 \\ 0 & 0 & 0 & 0 & 1 & -1 & 0 & 0 & 0 & 0 & 0 & 0 & 0 & 0 \\ 0 & 0 & 0 & 0 & 1 & 0 & -1 & 0 & 0 & 0 & 0 & 0 & 0 & 0 \\ 0 & 0 & 0 & 0 & 0 & 0 & 0 & 0 & 1 & 1 & 1 & 0 & 0 & 0 \\ 0 & 0 & 0 & 0 & 0 & 0 & 0 & 0 & 0 & 0 & 0 & 1 & 1 & 1 \\ 0 & 0 & 0 & 0 & 0 & 0 & 1 & 0 & 0 & 0 & 0 & 0 & 0 & -Z_{L}\end{array}\right]\left|\begin{array}{c}U_{S} \\ U_{1} \\ U_{2} \\ U_{3} \\ U_{4} \\ U_{5} \\ U_{6} \\ i_{S} \\ i_{1} \\ i_{2} \\ i_{3} \\ i_{4} \\ i_{5} \\ i_{6}\end{array}\right|=\left|\begin{array}{c}0 \\ 0 \\ 0 \\ 0 \\ 0 \\ 0 \\ 0 \\ 0 \\ 0 \\ 0 \\ 0 \\ 0 \\ 0\end{array}\right|$

where $a_{i}=d_{i}=\operatorname{ch}\left(r_{i} l_{i}\right), b_{i}=Z_{C} \operatorname{sh}\left(r_{i} l_{i}\right)$, and $c_{i}=\operatorname{sh}\left(r_{i} l_{i}\right) / Z_{C}$, $(i=1,2) ; Z_{S}$ is the source impedance, and $Z_{L}$ is the load impedance.

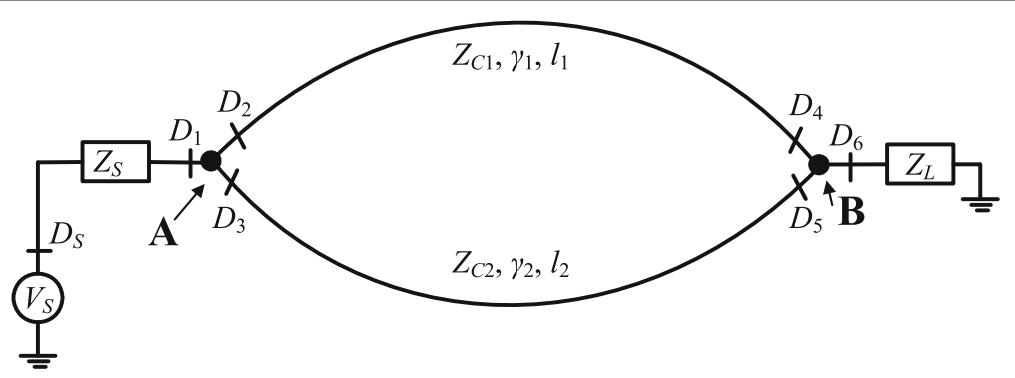

Fig. 5 A simple network with a ring topology 
Thus, the voltage transmission characteristic between the signal transmitter and a given receiver can be easily obtained. For example, the voltage transmission function between $D_{S}$ and $D_{6}$ in Fig. 5 is:

$$
H(f)=\frac{U_{6}}{U_{S}}=\frac{Z_{L}\left(a_{1} b_{2} d_{1}-b_{1} b_{2} c_{1}+a_{2} b_{1} d_{2}-b_{1} b_{2} c_{2}\right)}{\left(b_{1} b_{2}+\left(a_{1} b_{2}+a_{2} b_{1}\right) Z_{S}+\left(b_{1} d_{2}+b_{2} d_{1}\right) Z_{L}\right.}
$$

A physical network with the topology in Fig. 5 was established for the laboratory test, RG316-type coaxial cables and a HP8753D network analyzer. The parameters of the coaxial cable are $C_{0}=96 \mathrm{pF} / \mathrm{m}, L_{0}=276$ $\mathrm{nH} / \mathrm{m}, R_{0}=1 \mathrm{~m} \Omega / \mathrm{m}, G_{0}$ is neglected, and the lengths of $l_{1}$ and $l_{2}$ are set to $10 \mathrm{~m}$. The cables and interfaces and part of the physical configuration of the circuit are shown in Figs. 6 and 7, respectively. Figure 8 illustrates an actual waveform from the network analyzer.

The HP8753D network analyzer can easily determine the voltage transfer characteristic of networks with different topologies. The resulting curve is shown on its screen, as shown in Fig. 8, and the data can be easily stored and read using a floppy disk.

Figures 9 and 10 show the amplitude- and phasefrequency response characteristics of the voltage transfer function Eq. (10). The actual cable length and the length used in the simulation are not exactly identical, and the neglected cable connection resistance in simulation contributes to the major mismatch in these two figures.

\section{Simulation and laboratory test results for a network with a ring topology (Results and discussion 2)}

A network with a ring topology (Fig. 11) is used to analyze all factors that may affect the amplitude-frequency characteristic of the network. In this network, all power lines are of identical material with the following parameters: $C_{0}=$
$96 \mathrm{pF} / \mathrm{m}, L_{0}=276 \mathrm{nH} / \mathrm{m}, \quad R_{0}=1 \mathrm{~m} \Omega / \mathrm{m}$; and $G_{0}$ is neglected. Both source internal impedance $Z_{S}$ and load impedance $Z_{L}$ are $50 \Omega$; both branch loads $Z_{L 1}$ and $Z_{L 2}$ are $0 \Omega$, i.e., both are short-circuited. In the following, the simulation results of the amplitude-frequency characteristic are obtained using the proposed method, whereas the laboratory test results are measured using the HP8753D network analyzer on a real network.

\subsection{Changes in trunk length}

First, the effect of the trunk length on the amplitudefrequency transmission characteristics is considered. The trunk lengths (i.e., $l_{1}, l_{2}, l_{3}$, and $l_{4}$ in Fig. 11) are identical, and simulations and tests are performed with lengths of $10,20,30$, and $40 \mathrm{~m}$, whereas the lengths of the branch lines (i.e., $l_{5}$ and $l_{6}$ in Fig. 11) are held constant at $10 \mathrm{~m}$.

Figure 12 presents the amplitude-frequency characteristics of the voltage transfer function between load $Z_{L}$ and signal source $\mathrm{V}_{\mathrm{S}}$.

Figure 12 illustrates that the peaks in the amplitudefrequency characteristics fluctuate when the trunk length increases, whereas the locations and amplitudes of the notches do not change. The notches occur at the frequencies of 10, 20, and $30 \mathrm{MHz}$.

\subsection{Changes in branch length}

Next, the effect of the branch length on the amplitudefrequency transmission characteristic is simulated and tested. In Fig. 11, we maintained a constant trunk length of $l_{1}=l_{2}=l_{3}=l_{4}=10 \mathrm{~m}$, whereas the branch lengths $l_{5}$ and $l_{6}$ were changed to $10 \mathrm{~m}, 20 \mathrm{~m}, 30 \mathrm{~m}$, and $40 \mathrm{~m}$, respectively.

Figure 13 presents the amplitude-frequency characteristic of the voltage transfer function between load $Z_{L}$ and signal source $\mathrm{V}_{\mathrm{S}}$. We can learn that the numbers of peaks and notches in the amplitude-frequency characteristic curve increase when the branch length increases

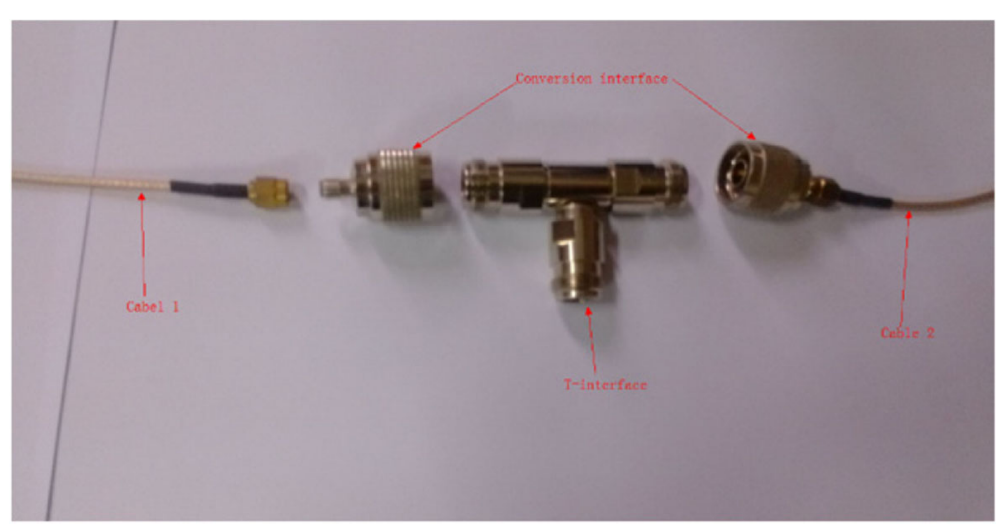

Fig. 6 The elements used 


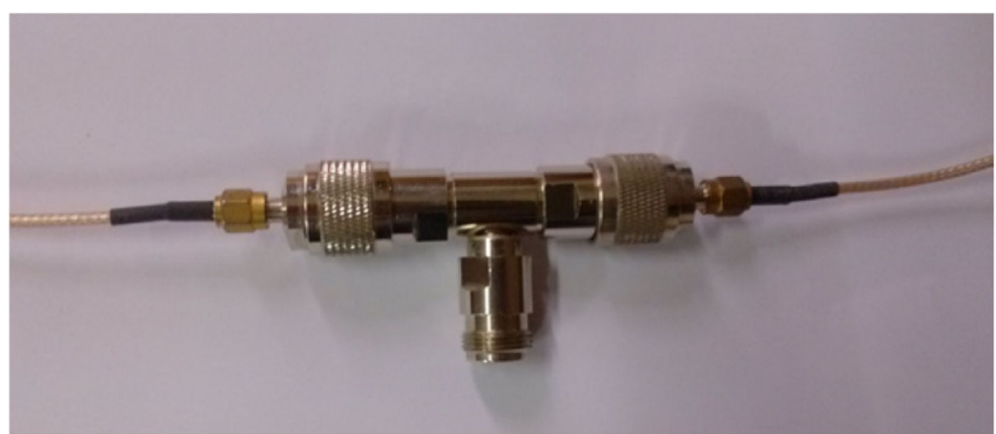

Fig. 7 A part of the lab test circuit

(the numbers of notches in Figs. 13a, b, c, and d are 3, 6, 9 , and 12 , respectively).

\subsection{Changes in the number of branches}

Next, we consider a network with the ring topology in Fig. 14. Length $l$ of all lines between adjacent nodes is $10 \mathrm{~m}$; thus, when the number of branches is $2,4,6$, or 8 , the length of $\mathrm{ADEB}$ and $\mathrm{AFHB}$ is $20,30,40$, or $50 \mathrm{~m}$, respectively. All branch loads (i.e., $Z_{L 11}, Z_{L 12}, \ldots, Z_{L n 1}, Z_{L n 2}$ ) are open circuit, and the other parameters are identical to those in Fig. 11.

Figure 15 shows the amplitude-frequency characteristic of the voltage transfer function between load $Z_{L}$ and signal source $V_{S}$. The peaks of the amplitude-frequency characteristic slightly fluctuate when the branch number increases. There are only 3 notches for various numbers of branches, but the amplitude of the notches increases with an increasing number of branches.

\section{Simulation and laboratory test results for a network with a mesh topology (Results and discussion 3)}

A network with a mesh topology (Fig. 14) is used to analyze all factors that may affect the amplitude-frequency characteristic of the network. In Fig. 14, all power lines are of identical material, and the following parameters are used: $C_{0}=96 \mathrm{pF} / \mathrm{m}, L_{0}=276 \mathrm{nH} / \mathrm{m}, R_{0}=1 \mathrm{~m} \Omega / \mathrm{m}$, and $G_{0}$ is neglected. Source internal impedance $Z_{S}$ and load impedance $Z_{L}$ are $50 \Omega$, and branch loads $Z_{L 1}$ and $Z_{L 2}$ are 0 $\Omega$. In the following, simulation results of the amplitude frequency characteristics are obtained using the proposed method, and the laboratory test results are measured using a network analyzer on a real network with the topology in Fig. 16.

\subsection{Changes in front and rear trunk length}

First, we maintain a constant trunk length of $l_{5}=l_{6}=$ $l_{7}=l_{8}=10 \mathrm{~m}$, a constant branch length of $l_{2}=l_{4}=10$ $\mathrm{m}$, and a constant link branch length of $l_{9}=20 \mathrm{~m}$. We change the front and rear trunk lengths $\left(l_{1}\right.$ and $l_{3}$, respectively) to $10,20,30$, and $40 \mathrm{~m}$.

Figure 17 shows the amplitude-frequency characteristic of the voltage transfer function between load $Z_{L}$ and signal source $V_{S}$. The amplitude-frequency characteristics vary only slightly for different front and rear trunk lengths; thus, this factor is not considered in the following analysis.

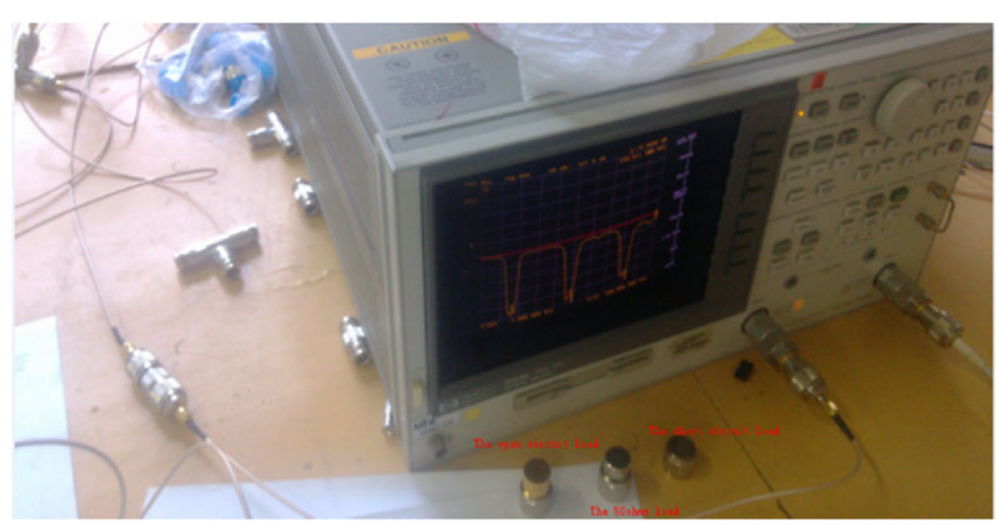

Fig. 8 The HP8753D network analyzer 


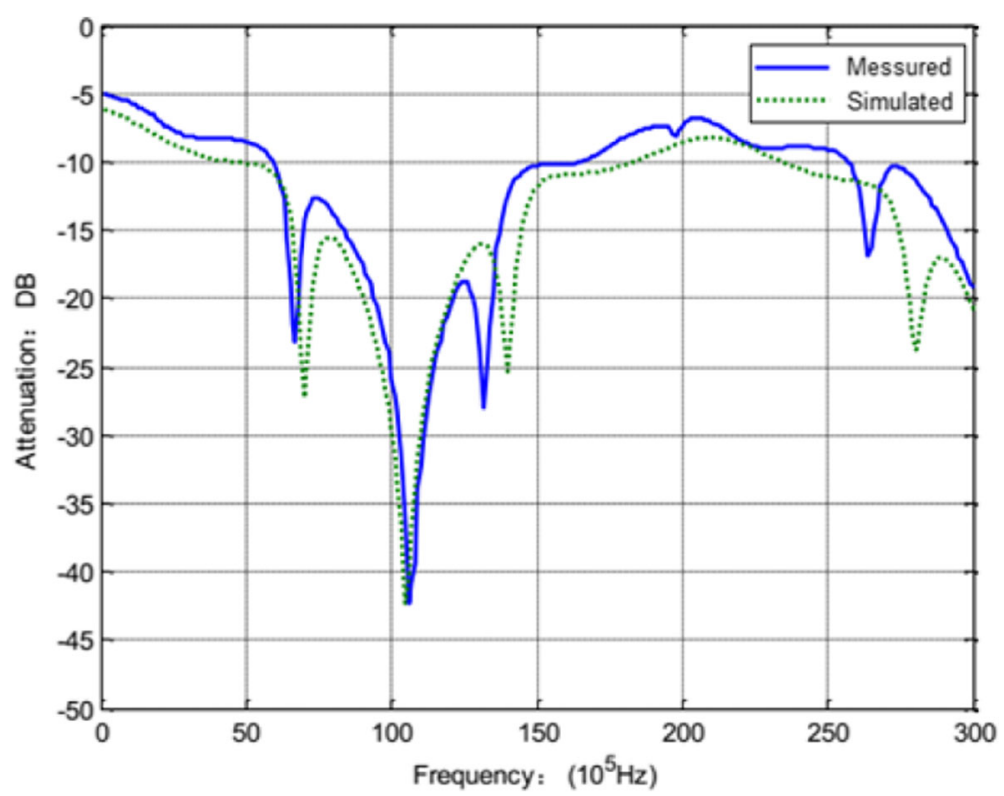

Fig. 9 Laboratory test and simulation results of amplitude-frequency characteristic

\subsection{Changes in branch length}

In Fig. 16, we maintain a constant front and rear trunk length of $l_{1}=l_{3}=10 \mathrm{~m}$, a trunk length of $l_{5}=$ $l_{6}=l_{7}=l_{8}=5 \mathrm{~m}$, and a link branch length of $l_{9}=10$ $\mathrm{m}$; we change the branch lengths $l_{2}$ and $l_{4}$ to 5,10 , 15 , and $20 \mathrm{~m}$.

Figure 18 shows the amplitude-frequency characteristic of the voltage transfer function between load $Z_{L}$ and signal source $\mathrm{V}_{\mathrm{S}}$.
The branch length significantly affects the amplitudefrequency characteristics; specifically, additional notches appear when the branch length is increased.

\subsection{Changes in trunk length}

In Fig. 16, we maintain constant front and rear trunk lengths of $l_{1}=l_{3}=10 \mathrm{~m}$, a branch length of $l_{2}=l_{4}=5 \mathrm{~m}$, and a link branch length of $l_{9}=10 \mathrm{~m}$. We vary the trunk lengths $l_{5}, l_{6}, l_{7}$, and $l_{8}$ to $5,10,15$, and $20 \mathrm{~m}$.

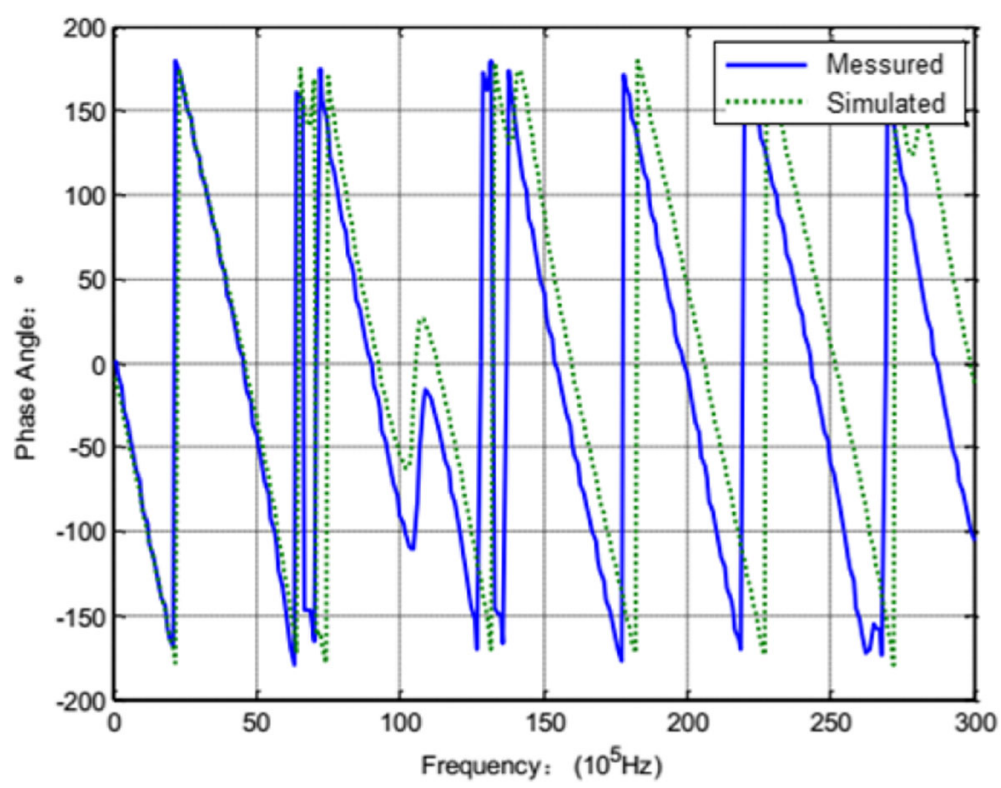

Fig. 10 Laboratory test and simulation results of phase-frequency characteristic 


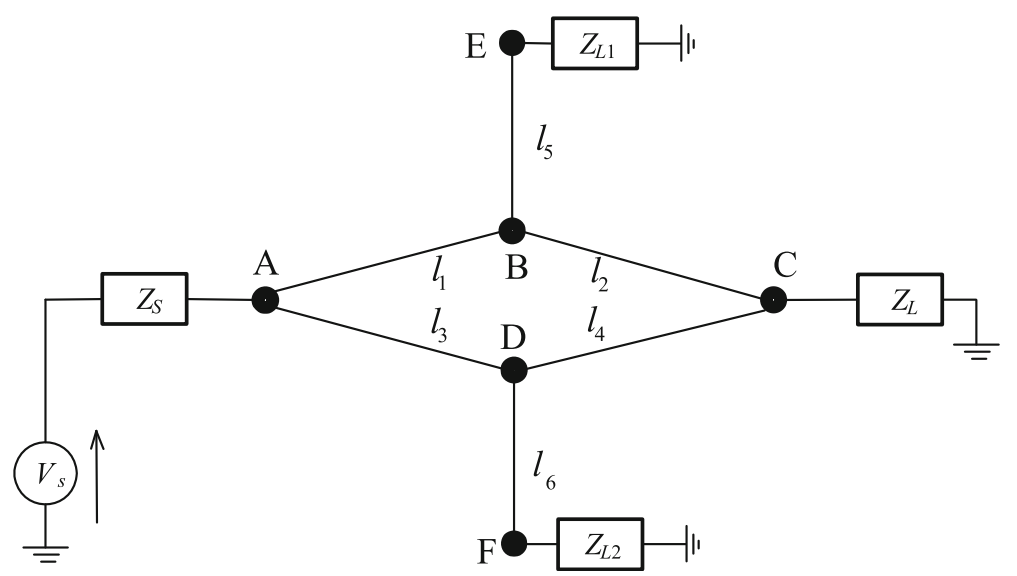

Fig. 11 Simple ring topology with branches

Figure 19 presents the amplitude-frequency characteristics of the voltage transfer function between load $Z_{L}$ and signal source $\mathrm{V}_{\mathrm{S}}$.

The trunk length has a complex effect on the transmission characteristics. As the trunk length increases, the number of notches change to different numbers in an alternating manner, e.g., 2 notches are present in Fig. 19a and c, whereas 4 notches are present in Fig. 19b and d.

\subsection{Proportional changes in the mesh length}

In Fig. 16, we maintain constant front and rear trunk lengths of $l_{1}=l_{3}=10 \mathrm{~m}$, whereas the trunk length $l_{5}=l_{6}=l_{7}=l_{8}$ is varied to $5,10,15$, and 20 $\mathrm{m}$, the branch length $l_{2}=l_{4}$ is proportionally changed to $5,10,15$, and $20 \mathrm{~m}$, and the link branch length $l_{9}$ is proportionally changed to $10,20,30$, and $40 \mathrm{~m}$.
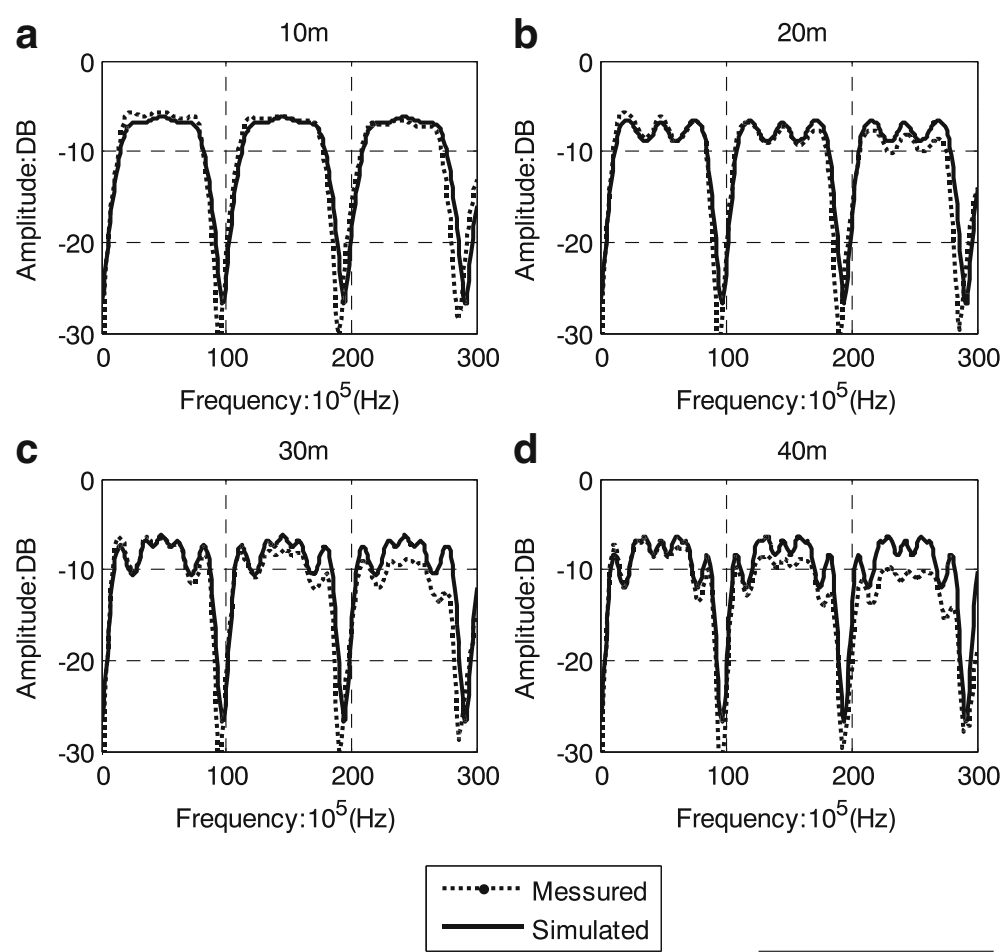

Fig. 12 Voltage transmission amplitude-frequency characteristic for various trunk lengths (a) $10 \mathrm{~m}$ (b) $20 \mathrm{~m}$ (c) $30 \mathrm{~m}$ (d) $40 \mathrm{~m}$ 

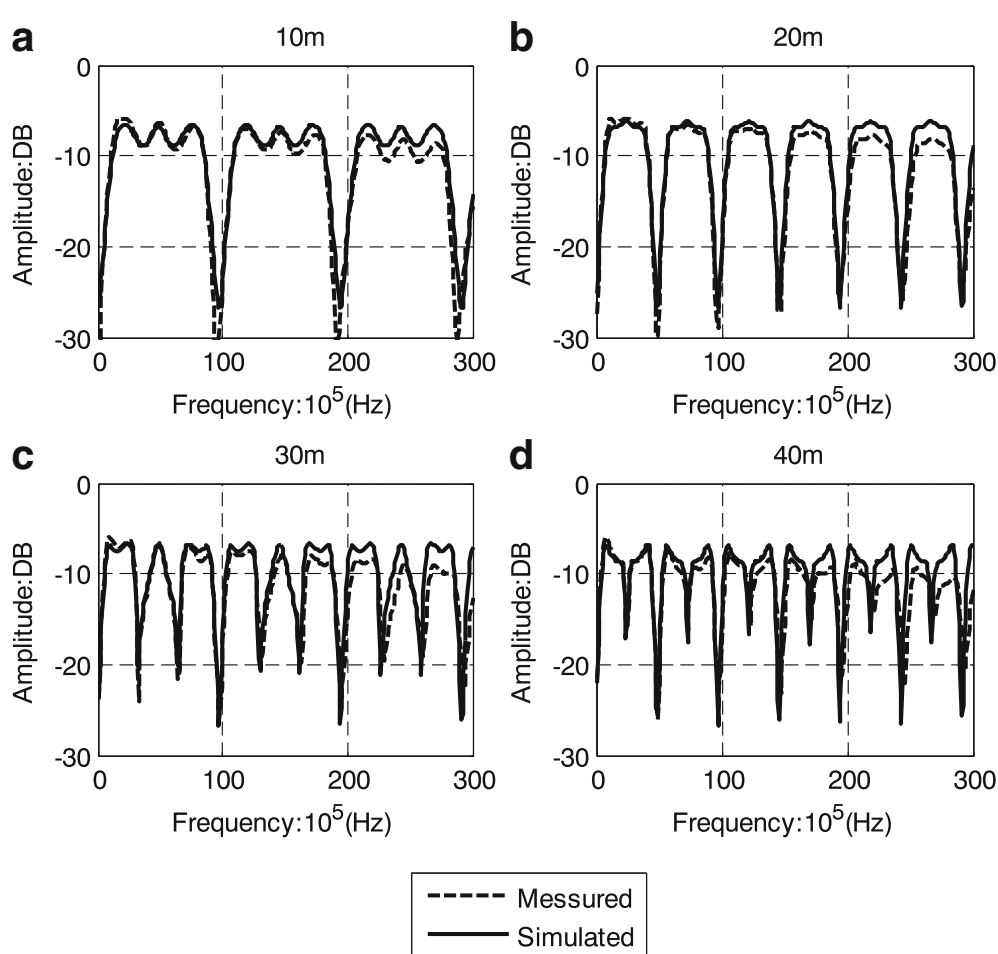

Fig. 13 Voltage transmission amplitude-frequency characteristic for various branch lengths (a) $10 \mathrm{~m}$ (b) $20 \mathrm{~m}$ (c) $30 \mathrm{~m}$ (d) $40 \mathrm{~m}$

Figure 20 shows the amplitude-frequency characteristics of the voltage transfer function between load $Z_{L}$ and signal source $\mathrm{V}_{\mathrm{S}}$.

The numbers of peaks and notches of the amplitudefrequency characteristics increase with the increase in line length of the mesh topology: the number of notches in Figs. 20a, b, c and d is 2, 4, 6, and 8, respectively.

If the frequency characteristics of the elements in the net can be accurately obtained, the proposed method can be used in a notably wide frequency range. Considering the current accuracy of the frequency characteristics, the requirements of high-speed communication in the PLC in future, and the restrictions of the frequency in simulation and lab test, the proposed method can be used in the range of $1-40 \mathrm{MHz}$.

\section{Conclusions}

A novel channel modeling method based on the information node that is universally applicable to various topologies that may emerge in smart distribution grids in the future is present in this paper. The applications of this method in a simple ring network and a complex

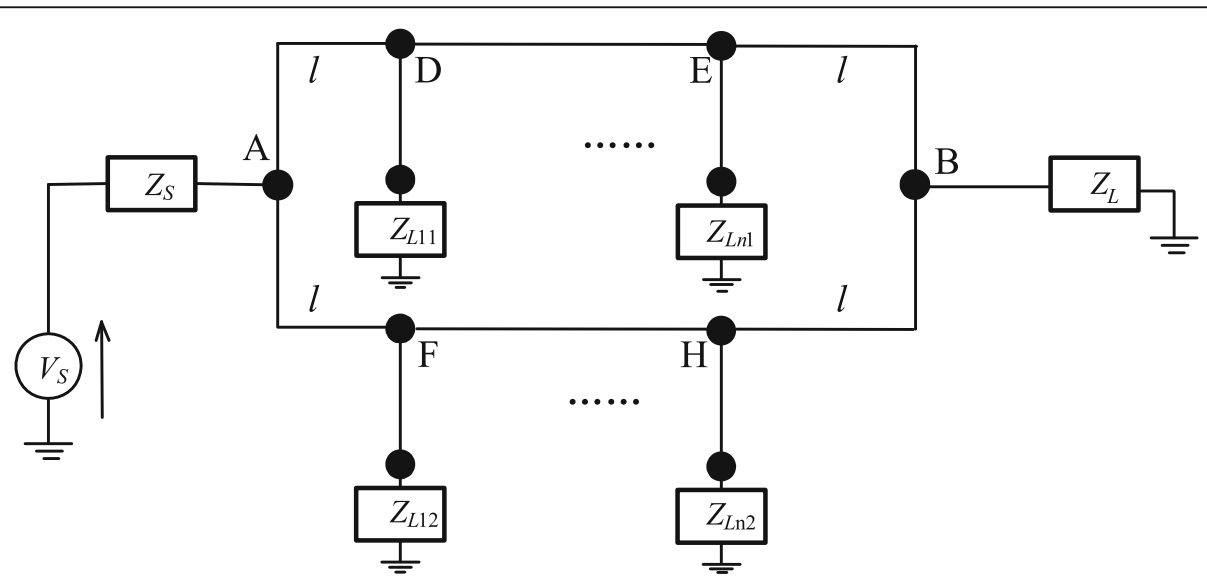

Fig. 14 Ring topology with multiple branches 

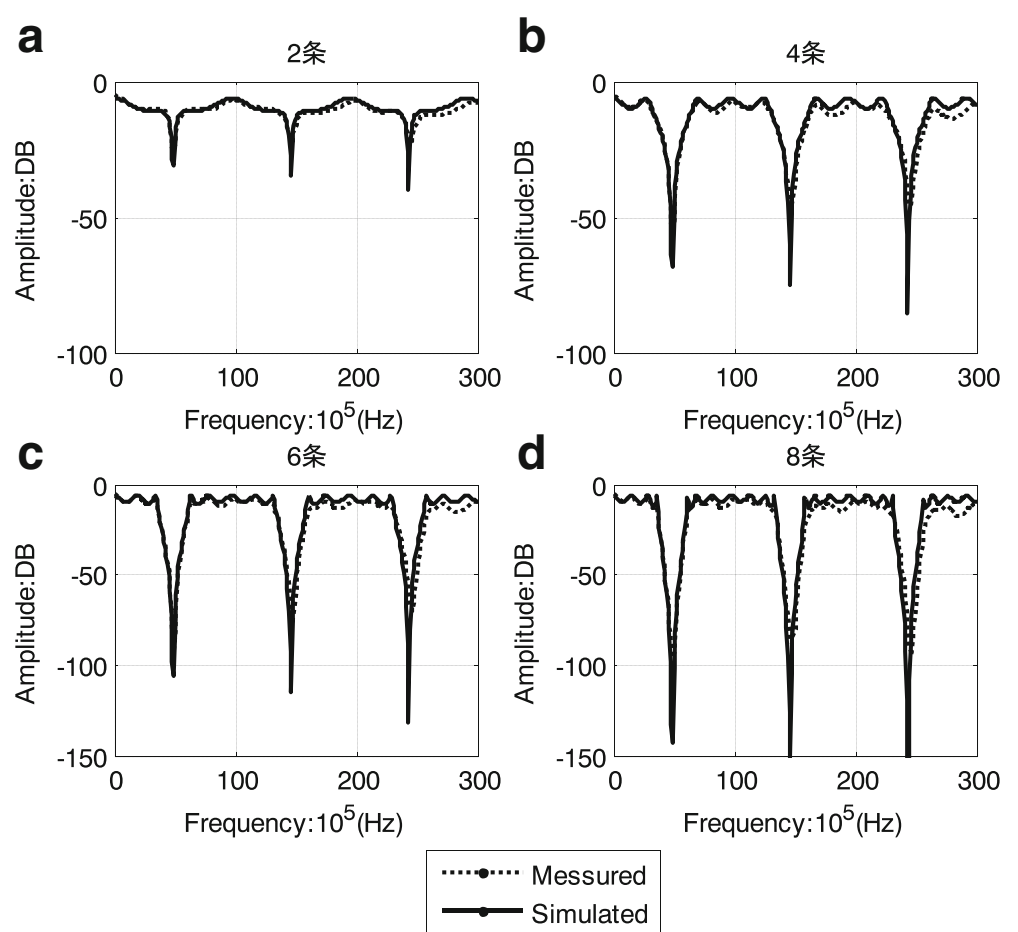

Fig. 15 Voltage transmission amplitude-frequency characteristic for various numbers of branches (a) 2 branches (b) 4 branches (c) 6 branches (d) 8 branches

mesh network show that the proposed method has significant advantages over the traditional modeling method, such as clear physical concepts, simple calculations, and ease of implementation with conventional computational software. Based on the proposed method, several meaningful conclusions regarding the influence laws for the channel characteristics of network with ring or mesh topology can be easily drawn, such as that the trunk length has little effect on the transfer characteristic, while the proposed modeling method and the analysis results provide the necessary theoretical basis and technical measure for the design of PLC modulation method for a smart distribution grids.

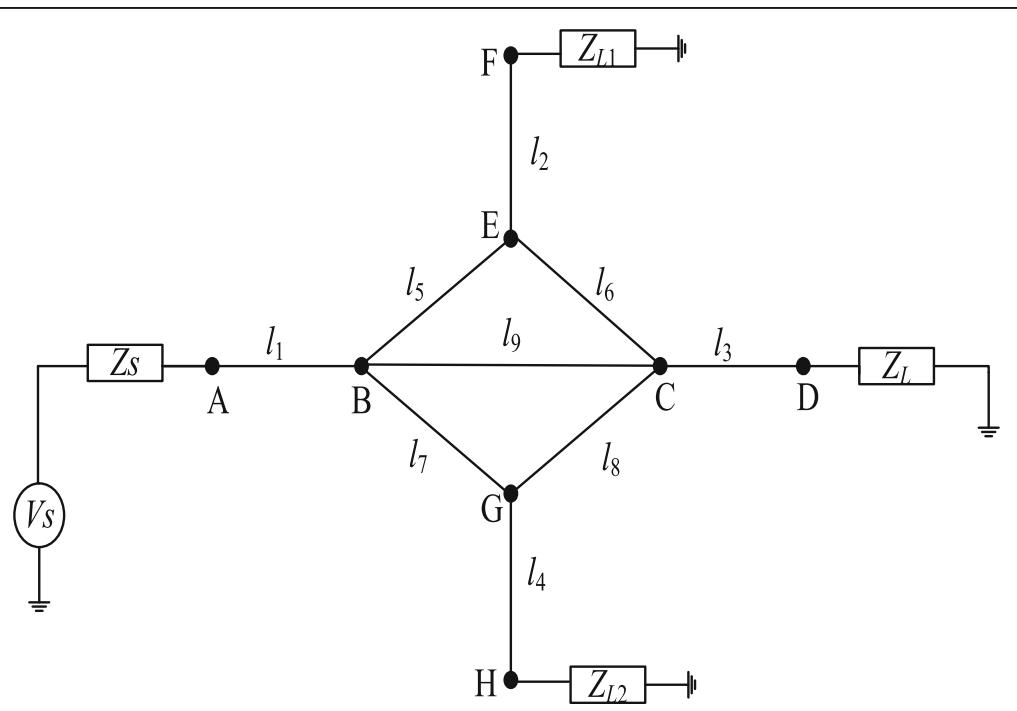

Fig. 16 Simple network with a mesh topology 

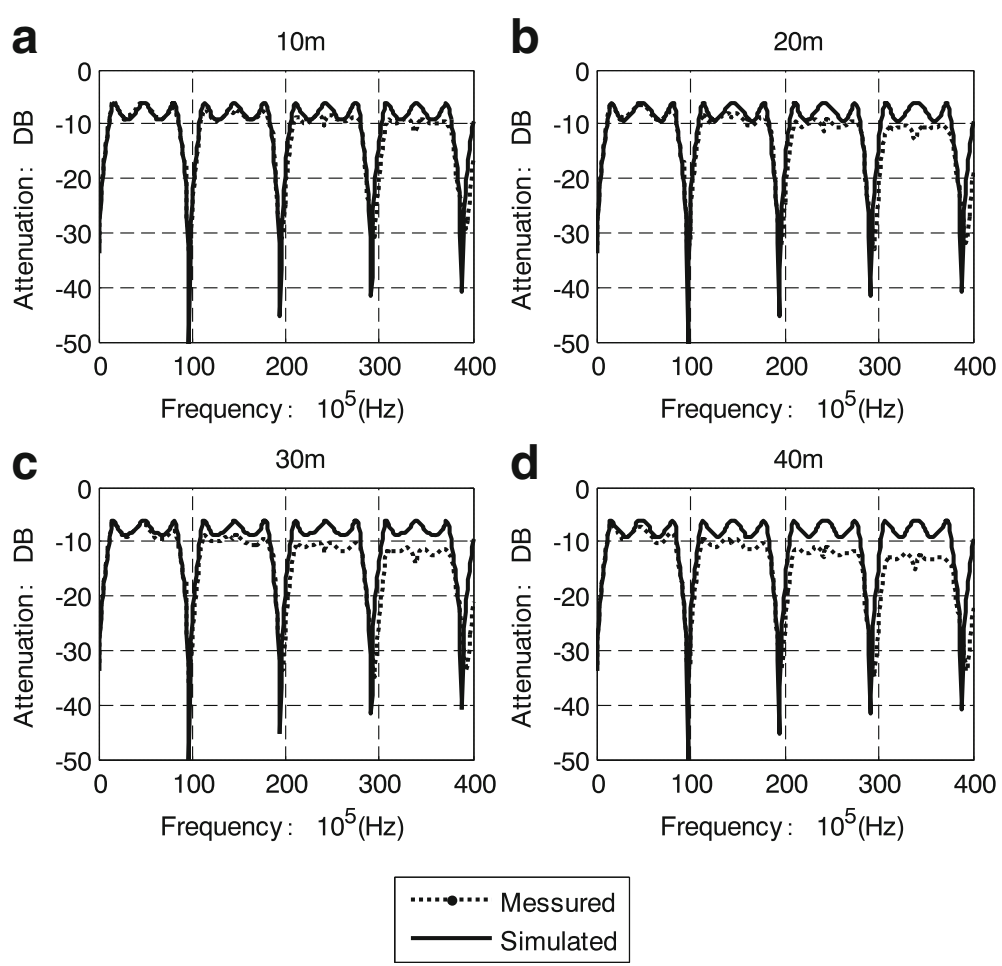

Fig. 17 Voltage transmission amplitude-frequency characteristic for various front and rear trunk lengths (a) $10 \mathrm{~m}$ (b) $20 \mathrm{~m}$ (c) $30 \mathrm{~m}$ (d) $40 \mathrm{~m}$
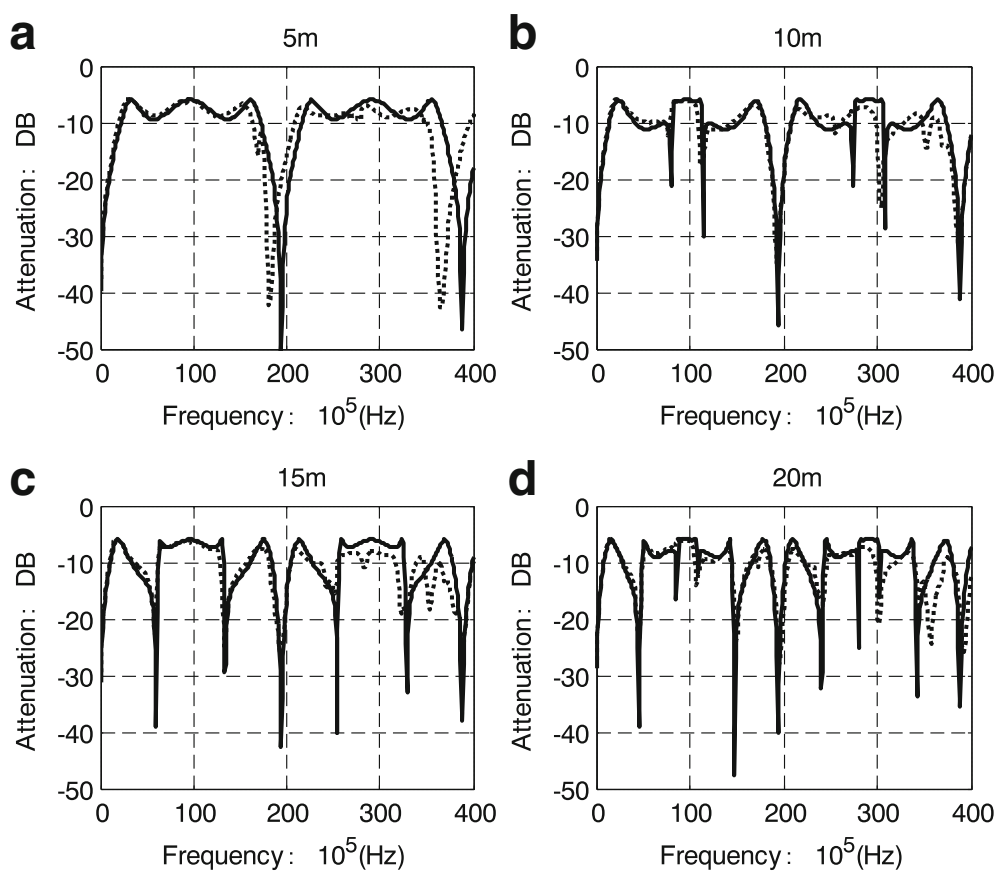

\section{Messured}

Simulated

Fig. 18 Voltage transmission amplitude-frequency characteristic for various branch lengths (a) $5 \mathrm{~m}$ (b) $10 \mathrm{~m}$ (c) $15 \mathrm{~m}$ (d) $20 \mathrm{~m}$ 

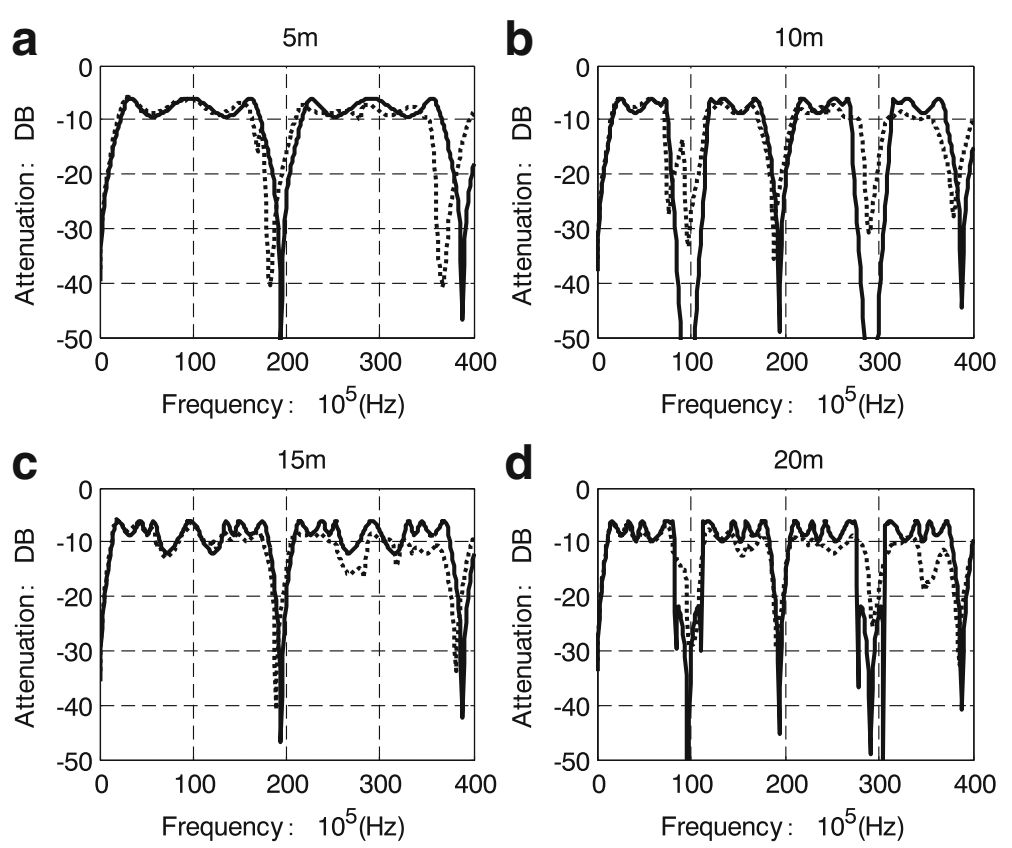

$$
\text { Simulated }
$$

Fig. 19 Voltage transmission amplitude-frequency characteristic for various trunk lengths (a) $5 \mathrm{~m}$ (b) $10 \mathrm{~m}$ (c) $15 \mathrm{~m}(\mathbf{d}) 20 \mathrm{~m}$
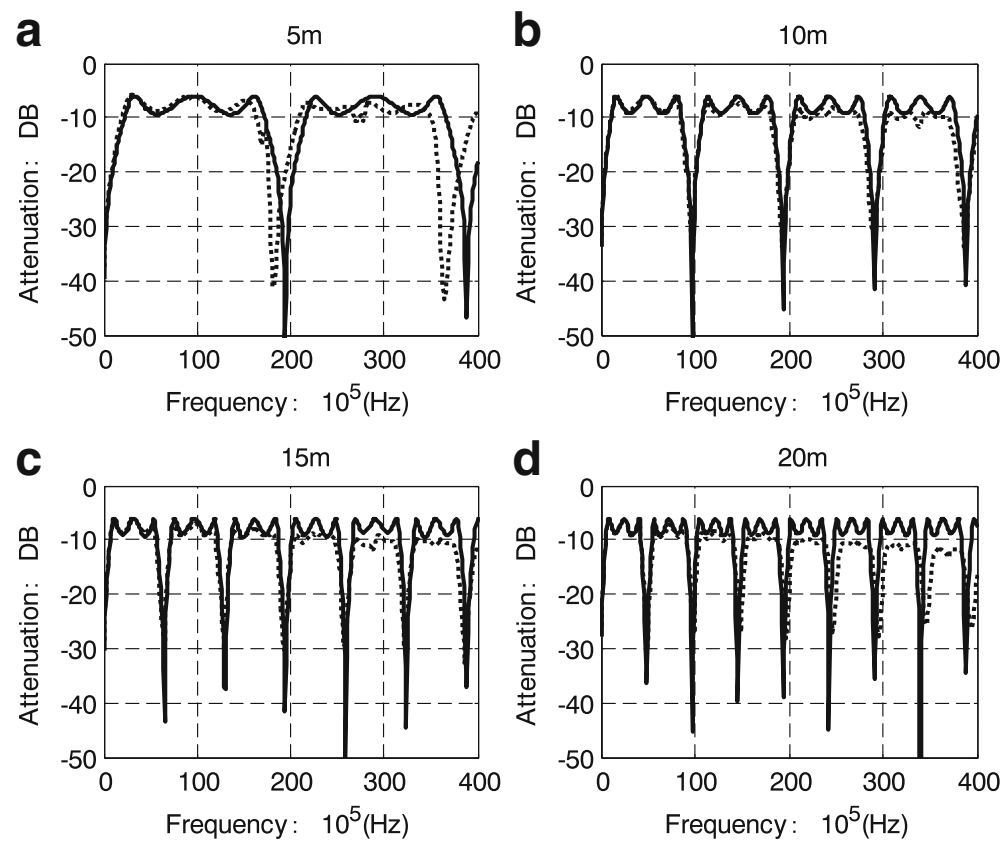

Messured

Simulated

Fig. 20 Voltage transmission amplitude-frequency characteristic with proportional changes in the line length of the mesh topology (a) $5 \mathrm{~m}$ (b) $10 \mathrm{~m}$ (c) $15 \mathrm{~m}$ (d) $20 \mathrm{~m}$ 


\section{Authors' contributions}

$J L$ carried out the studies about the PLC channel modeling theory, participated in the sequence alignment and drafted the manuscript. CW carried out the simulations of the proposed modeling method. WZ and Y-YL carried out the experimental tests and performed the statistical analysis of the results. WC built the theory model of the PLC channel modeling method. All authors read and approved the final manuscript.

\section{Authors' information}

Jian Le was born in Huanggang, Hubei, China in 1975. Correspondence author. He is currently Associate Professor with the college of electrical engineering, $\mathrm{WHU}$, where he has been working on flexible ac transmission technology and power quality control technology.

Cao Wang was born in Bengbu, Anhui, China in 1995. He received his B.S. degree from the College of Energy and Electrical Engineering at Wuhan University (WHU), Wuhan, China, in 2016. He is now working towards a Master degree in electrical engineering at Wuhan University. He has been researching power quality and its control technology.

Wu Zhou was born in Xiantao, Hubei, China in 1992. He received his B.S. degree from the college of electrical engineering at Hohai University (HHU), Nanjing, China, in 2014. He is currently working towards a Master degree in electrical engineering at Wuhan University. He has been working on research of optimal operation and control in power system.

Yong-yan Liu was born in Changsha, Hunan, China in 1992. She received her B. S. degree from the college of electrical and information engineering at Hunan University (HNU), Changsha, China, in 2014. She is currently working towards a Mater degree in electrical engineering at Wuhan University. She has been working on research of distributed power operation and its control technology.

Wei Cai was born in Qian jiang, Hubei, China in 1987. He received his B.S. and Ph.D degree from the college of electrical engineering at Wuhan University (WHU), Wuhan, China, in 2008 and 2013 respectively. He has been working as engineer within State Grid Electric Power Research Institute, Wuhan, China and has worked on power system operation.

\section{Competing interests}

There is no conflict of interest in the submission of this manuscript, and the manuscript is approved by all authors for publication.

Author details

${ }^{1}$ School of Electrical Engineering, Wuhan University, Wuhan 430072, China.

${ }^{2}$ State Grid Electric Power Research Institute, Wuhan 430074, China.

Received: 3 December 2016 Accepted: 10 March 2017

Published online: 17 April 2017

\section{References}

1. Moya, S., Hadad, M., Funes, M., et al. (2016). Broadband PLC-channel equalisation in the frequency domain based on complementary sequences. IET Communications, 10(13), 1605-13.

2. Chen, C., Chen, Y., Ding, N., et al. (2012). Accurate sampling timing acquisition for baseband OFDM power-line communication in non-gaussian noise. IEEE Transactions on Communications, 61(4), 1608-20.

3. Pagani, P., \& Schwager, A. (2016). A statistical model of the in-home MIMO PLC channel based on European field measurements. IEEE Journal on Selected Areas in Communications, 34(7), 2033-44.

4. Adebisi, B., Treytl, A., Haidine, A., et al. (2011). IP-centric high rate narrowband PLC for smart grid applications. IEEE Transactions On Communications Magazine, 49(12), 46-54.

5. Ningzhe, X., Sidong, Z., Yue, S., et al. (2016). PLC-oriented access point location planning algorithm in smart-grid communication networks. China Communications, 13(9), 91-102.

6. Galli, S., Scaglione, A., \& Wang, Z. (2011). For the grid and through the grid: The role of power line communications in the smart grid. IEEE Proceedings, 99(6), 998-1027.

7. Obermeier, M., Braun, S., \& Vogel-Heuser, B. (2015). A model-driven approach on object-oriented PLC programming for manufacturing systems with regard to usability. IEEE Transactions on Industrial Informatics, 11(3), 790-800.

8. Cataliotti, A., Cara, D. D., Fiorelli, R., et al. (2012). Power-Line communication in medium-voltage system: simulation model and onfield experimental tests. IEEE Transactions on Power Delivery, 27(1), 62-9.
9. Tonello, A. M., Versolatto, F., Bejar, B., et al. (2012). A fitting algorithm for random modeling the PLC channel. IEEE Transactions on Power Delivery, 27(3), 1477-84.

10. Shlezinger, N., \& Dabora, R. (2015). On the capacity of narrowband PLC channels. IEEE Transactions on Communications, 63(4), 1191-201.

11. Francisco, J., Pacheco, S., Sotorrío-Ruiz, P. J., et al. (2014). PLC-based PV plants smart monitoring system: field measurements and uncertainty estimation. IEEE Transactions on Instrumentation and Measurement, 63(9), 2215-22.

12. Gianaroli, F., Pancaldi, F., \& Vitetta, G. M. (2013). The impact of load characterization on the average properties of statistical models for powerline channels. IEEE Transactions on Smart Grid, 4(2), 677-85.

13. Galli, S., \& Banwell, T. C. (2006). A deterministic frequency-domain model for the indoor power line transfer function. IEEE Journal on Selected Areas in Communications, 24(7), 1304-16.

14. Rennane, A., Konate, C., \& Machmoum, C. M. (2008). A simplified deterministic approach for accurate modeling of the indoor power line channel. IEEE Conf. On Systems and Networks Communications, Malta, 2008, 121-6.

15. Ricard, A., Miquel, R., Joan, R. R., et al. (2009). Scattering parameters-based channel characterization and modeling for underground medium-voltage power-line communications. IEEE Transactions on Power Delivery, 24(3), 1122-31.

16. Zhiyuan, X., Yan, S., \& Yihe, G. (2010). Modeling and analysis of medium voltage power line channel base on transmission line theory. Electric Power Science and Engineering, 26(7), 5-8.

17. Mannah, M. A. Ginot, N. \& Batard, C. (2014). Effect of the power cable on data transmission over a pulsewidth-modulated network. IEEE Transactions on Industrial Electronics, 61(8), 4238-45.

18. Meng, H., Chen, S., Guan, Y. L., et al. (2004). Modeling of transfer characteristics for the broadband power line communication channel. Power Delivery. IEEE Transactions on Power Delivery, 19(3), 1057-64.

19. Cai, W., Le, J., \& Liu, K. P. (2013). The analysis of the indoor PLC channel characteristics based on information nodes channel modeling method. International Journal of Computer \& Electrical Engineering, 5(2), 155-9.

\section{Submit your manuscript to a SpringerOpen ${ }^{\circ}$ journal and benefit from:}

- Convenient online submission

- Rigorous peer review

- Immediate publication on acceptance

- Open access: articles freely available online

- High visibility within the field

- Retaining the copyright to your article

Submit your next manuscript at $>$ springeropen.com 